\title{
氢自由基引发的 1,2-炔基迁移
}

\author{
赵琦屠树江* 姜波* \\ (江苏师范大学化学与材料科学学院 徐州 221116)
}

\begin{abstract}
摘要 报道了一类新颖的 $\mathrm{Fe}(\mathrm{III})$ 介导的形式上的非活性烯烃的氢炔化反应. 该反应利用 $\mathrm{Fe}(\mathrm{acac})_{3}$ 与苯硅烷反应能原位 产生氢自由基的特性实现了氢自由基诱导的 1,4-烯炔的分子内 1,2-炔基迁移, 合成了一系列 $\alpha$-炔酮类化合物, 产率中等 到良好. 基于实验结果及文献报道, 提出了可能的反应机理, 其涉及氢自由基加成、3-exo-dig 环化(反鲍德温规则)和 $\mathrm{C}-\mathrm{C}$ 键的断裂重组等. 此外, 该反应具有良好的官能团兼容性, 如雌酮衍生的 1,4-烯炔也能成功参与该反应.
\end{abstract}

关键词 非活性烯烃氢炔化; 鲍德温规则; 炔基迁移; 1,4-烯炔; $\alpha$-炔酮衍生物

\section{Hydrogen Radical Initiated 1,2-Alkynyl Migration \\ Zhao, Qi Tu, Shu-Jiang* Jiang, Bo* \\ (School of Chemistry and Material Science, Jiangsu Normal University, Xuzhou 221116)}

\begin{abstract}
As inexpensive and readily available feedstocks, alkenes possess a unique reactivity profile and thus have been extensively applied in synthetic chemistry. Specifically, radical-triggered difunctionalization of alkenes provides a valuable synthetic strategy for their high utilization by incorporating two functional groups across the $\mathrm{C}=\mathrm{C} \pi$ system. Despite the great achievements gained in this field, the vast majority of well-developed methods generally focus on activated alkenes, because its nascent alkyl radical needs to be stabilized by adjacent functional groups (e.g. aryl, carbonyl, heteroatom) via $p-\pi$ conjugate effect. However, radical induced difunctionalization of unactivated alkenes remains elusive. Herein, a new protocol for $\mathrm{Fe}(\mathrm{III})$ mediated hydroalkynylation of unactivated olefins is reported. By using the characteristics of the in-situ-generated hydrogen radical from the interaction of $\mathrm{Fe}(\mathrm{acac})_{3}$ and phenylsilane, hydrogen radical-triggered intramolecular 1,2-alkynyl migration was realized in this reaction, which led to the synthesis of a series of $\alpha$-alkynyl ketones with moderate to good yields. Based on the experimental results and literature reports, a reasonable reaction mechanism was proposed, which involved hydrogen radical addition, 3-exo-dig cyclization (anti-Baldwin rules) and $\mathrm{C}-\mathrm{C}$ bond breaking/recombination. Moreover, the reaction features good tolerance of functional groups, in which estrone-derived 1,4-enyne could be accommodated. A typical procedure for hydroalkynylation of unactivated alkenes is as follows: $\mathrm{Fe}(\mathrm{acac})_{3}(1.2$ equiv., $0.24 \mathrm{mmol})$ and $\mathrm{Na}-$ $\mathrm{HCO}_{3}$ (1.0 equiv., $0.2 \mathrm{mmol}$ ) are added to the 10 -mL pressure tube. Then 1,4-enynes (1.0 equiv., $\left.0.2 \mathrm{mmol}\right)$ and phenylsilane (2.0 equiv., $0.4 \mathrm{mmol}$ ) are dissolved in $1.0 \mathrm{~mL}$ ethyl alcohol, respectively. Both of them are injected into this vial. The reaction system was sealed and stirred at $100{ }^{\circ} \mathrm{C}$ until the 1,4-enynes consumed that is determined by thin layer chromatography (TLC). After the reaction completes, the resulting mixture is extracted with EtOAc for three times, then the organic phase is concentrated and evaporated on a rotary evaporator. The residue was purified by chromatography on silica gel with petroleum ether/ethyl acetate $(V: V=75: 1)$ as the eluent to afford $\alpha$-alkynyl ketones.

Keywords hydroalkynylation of unactivated alkene; Baldwin rule; alkynyl migration; 1,4-enyne; $\alpha$-alkynyl ketone
\end{abstract}

\section{1 引言}

烯烃广泛地存在于各类有机化合物中，如天然产 物、药物以及功能材料等. 近年来, 烯烃的双官能化反 应为烯烃化合物的高效利用提供了一种有效的方法, 已 成为有机合成研究领域的热点之一. 虽然烯烃的双官能 化反应在过去的十几年中取得了长足的发展 ${ }^{[1]}$, 但主要 集中于活性烯烃的双官能化, 而非活性烯烃的双官能化 的研究相对滞后. 得益于可控自由基转化的迅速发展, 自由基诱导的非活性烯烃的双官能化反应研究引起了
化学工作者极大的关注, 取得了一些研究进展 ${ }^{[2]}$. 如利 用叔醇化合物参与的自由基引发的分子内官能团迁移 反应为非活性烯烃的双官能化提供了一类非常重要的 合成策略 ${ }^{[3]}$, 已实现了非活性烯烃的双官能化, 如芳基 化 ${ }^{[4]}$ 、氧基化 ${ }^{[5]} 、$ 亚胺化 ${ }^{[6]}$ 、甲酰基化 ${ }^{[7]}$ 、杂芳基化 ${ }^{[8]}$ 、 烯基化 ${ }^{[9]}$ 、炔基化 ${ }^{[10]}$ 等. 2017 年朱晨课题组 ${ }^{[100]}$ 首次通过 光氧化还原催化反应，实现了烯烃三氟甲基化引发的 1,4-和 1,5-炔基迁移反应. 他们利用理论计算研究反应 机理发现: 该反应过程经历 5 或 6-exo-dig 环化过渡态是

*E-mail: laotu@jsnu.edu.cn (SJT); jiangchem@jsnu.edu.cn (BJ)

Received April 30, 2019; published June 21, 2019.

Supporting information for this article is available free of charge via the Internet at http://sioc-journal.cn.

Project supported by the National Natural Science Foundation of China (No. 21871112) and the Qing Lan Project of Jiangsu Education Committee (No. QL2016006).

项目受国家自然科学基金(No. 21871112)和江苏省青蓝工程(No. QL2016006)资助. 
有利的, 而3-exo-dig 环化过渡态则是不利的, 这一现象 与 Baldwin 规则相符. 因此, 利用自由基诱导的 3-exo-dig 环化过渡态来实现分子内 1,2-炔基迁移是行不 通的 ${ }^{[11]}$. 最近, 我们课题组制备了烯烃上具有甲基的 1,4-烯炔合成子, 将其与环烷烃在 Fe/DTBP(二叔丁基过 氧化物)的催化氧化体系下进行自由基引发的加成-环 化反应，实现了反 Baldwin 规则的分子内 1,2-炔基迁 移 ${ }^{[12]}$. 这一有意义的结果促使我们进一步深入研究此 类反 Baldwin 规则迁移策略的普适性和各类自由基的兼 容性.

烯烃的氢炔化反应成为合成内炔的重要合成方法 之一 ${ }^{[13]}$, 其主要集中于活性烯烃 ${ }^{[14]}$, 如李必杰课题组 ${ }^{[15]}$ 报道了利用 $\alpha, \beta$-不饱和酰胺实现烯基的催化不对称氢炔 化反应. 樊保敏课题组 ${ }^{[14 \mathrm{~m}]}$ 首次利用铱催化末端炔烃与 降冰片烯反应, 实现了非活性烯烃的不对称氢炔化. 相 较而言, 非活性烯烃的氢炔化反应的报道相当少 ${ }^{[16]}$. 2007 年, Nishimura 和 Hayashi 等 ${ }^{[16 a]}$ 利用铑催化的炔醇 类化合物不对称 1,3-炔基迁移实现了非活性烯烃的氢炔 化反应. 尽管上述工作建立了一些有效的氢炔化反应, 但其种类仍相当有限. 因此, 发展新的、更易操作的非 活性烯烃的氢炔化的方法具有重要研究价值. 2014 年, Baran 课题组 ${ }^{[17]}$ 报道了 $\mathrm{Fe}$ (III) 介导的苯硅烷原位产生氢 自由基, 进而引发自由基串联反应, 实现了杂原子取代 烯烃与缺电子烯烃的交叉偶联反应. 此结果为实现烯烃 的氢化提供了新的反应模式, 并在此基础上, 相继实现 了烯烃的还原偶联、氢胺化、氢甲基化、氢烷基化、氢 烯丙基化、氢芳基化等 ${ }^{[18]}$. 其中, 崔孙良课题组 ${ }^{[19]}$ 利用 炔溴和非活性烯烃为原料, 在乙酰丙酮铁和苯硅烷的催 化氢化体系下, 实现了非活性烯烃的氢炔化反应. 基于 上述成功的转化和结合我们之前的工作, 本工作利用廉 价的乙酰丙酮铁 $\left[\mathrm{Fe}(\mathrm{acac})_{3}\right]$ 介导的苯硅烷原位产生氢自 由基，使其引发 1,4-烯炔衍生物经反 Baldwin 规则的 3-exo-dig 环化和分子内的 1,2-炔基迁移, 合成了一系列 的 $\alpha$-炔酮类化合物, 同时实现了形式上的非活性烯烃的 氢炔化(Scheme 1).

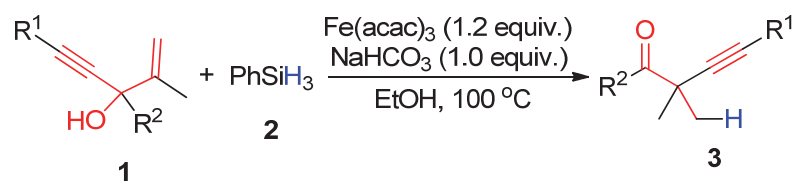

图式 1 自由基诱导的 $\alpha$-炔酮类化合物的合成

Scheme 1 Radical-induced synthesis of $\alpha$-alkynyl ketones

\section{2 结果与讨论}

\section{1 反应条件优化}

研究开始以 1,4-烯炔 $\mathbf{1 a}$ 作为反应底物, 苯硅烷 $\mathbf{2}$ 为 氢源试剂, 乙醇为溶剂, $\left[\mathrm{Fe}(\mathrm{acac})_{3}\right.$ ] 为催化剂, 在 $80{ }^{\circ} \mathrm{C}$ 下进行了反应尝试. 此反应能顺利进行, 但仅能得到
11\%产率的氢炔化产物 3a(表 1, Entry 1). 考虑到此反应 过程中涉及到脱质子过程，因此向反应体系中加入了碳 酸氢钠, 结果显示产物 3a 产率升到了 34\%(表 1, Entry $2)$. 随后对 $\mathrm{Fe}(\mathrm{acac})_{3}$ 的用量进行了篮选. 结果显示: 催 化量 $(10 \mathrm{~mol} \%)$ 的 $\mathrm{Fe}(\mathrm{acac})_{3}$ 明显抑制反应, 而增加其用 量则有利于该反应(表 1 , Entries 3,4). 利用碳酸钠代替 碳酸氢钠没有改善目标产物的产率(表 1, Entry 5). 升高 温度至 $100{ }^{\circ} \mathrm{C}$ 时，该反应以 $56 \%$ 的产率得到炔基迁移 产物 3a(表 1, Entry 6). 减少苯硅烷的用量, 产物 $\mathbf{3 a}$ 的收 率略有降低(表 1, Entry 7). 利用 $\mathrm{Co}(\mathrm{acac})_{3}$ 替代 $\mathrm{Fe}(\mathrm{acac})_{3}$ 则完全抑制了该反应过程(表 1, Entry 8). 降低 Fe(acac) 的用量不利于目标产物 3a 的形成(表 1, Entry 9). 增加其 用量至 1.2 equiv. 时, 可得到产物 3a, 产率为 64\%(表 1, Entry 10). 继续增加 $\mathrm{Fe}(\mathrm{acac})_{3}$ 的用量至 2.0 equiv. 时, 目 标产物 3a 的收率明显降低(表 1, Entry 11), 这可能由于 $\mathrm{Fe}(\mathrm{acac})_{3}$ 的用量过多时快速促进了副产物的形成而造 成的. 当使用氮气保护时, 目标产物 $\mathbf{3 a}$ 的收率仅为 $43 \%$ ，且有部分起始原料被回收(表 1, Entry 12), 这说明 反应体系中产生的部分 $\mathrm{Fe}(\mathrm{II})$ 物种被空气中的氧气氧化 成 $\mathrm{Fe}$ (III)物种继续促进该反应的转化. 因此, 确定了反 应的最优条件为 $\mathrm{Fe}(\mathrm{acac})_{3}$ (1.2 equiv.)、苯硅烷(2.0 equiv.)、碳酸氢钠(1.0 equiv.)、在 $100{ }^{\circ} \mathrm{C}$ 空气条件下进 行反应.

\section{表 1 反应条件优化 ${ }^{a}$}

Table 1 Optimization of the reaction conditions

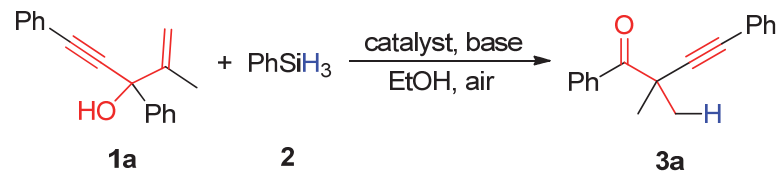

\begin{tabular}{|c|c|c|c|c|c|}
\hline Entry & Ratio (1a : 2a) & Base & Metal salt ( $x$ /equiv.) & Temp. $/{ }^{\circ} \mathrm{C}$ & Yield $^{b} / \%$ \\
\hline 1 & $1: 2$ & - & $\mathrm{Fe}(\mathrm{acac})_{3}(0.5)$ & 80 & 11 \\
\hline 2 & $1: 2$ & $\mathrm{NaHCO}_{3}$ & $\mathrm{Fe}(\mathrm{acac})_{3}(0.5)$ & 80 & 34 \\
\hline 3 & $1: 2$ & $\mathrm{NaHCO}_{3}$ & $\mathrm{Fe}(\mathrm{acac})_{3}(0.1)$ & 80 & 12 \\
\hline 4 & $1: 2$ & $\mathrm{NaHCO}_{3}$ & $\mathrm{Fe}(\mathrm{acac})_{3}(1.0)$ & 80 & 41 \\
\hline 5 & $1: 2$ & $\mathrm{Na}_{2} \mathrm{CO}_{3}$ & $\mathrm{Fe}(\mathrm{acac})_{3}(1.0)$ & 80 & 32 \\
\hline 6 & $1: 2$ & $\mathrm{NaHCO}_{3}$ & $\mathrm{Fe}(\mathrm{acac})_{3}(1.0)$ & 100 & 56 \\
\hline 7 & $1: 1.5$ & $\mathrm{NaHCO}_{3}$ & $\mathrm{Fe}(\mathrm{acac})_{3}(1.0)$ & 100 & 52 \\
\hline 8 & $1: 2$ & $\mathrm{NaHCO}_{3}$ & $\mathrm{Co}(\mathrm{acac})_{3}(1.0)$ & 100 & Trace \\
\hline 9 & $1: 2$ & $\mathrm{NaHCO}_{3}$ & $\mathrm{Fe}(\mathrm{acac})_{3}(0.8)$ & 100 & 55 \\
\hline 10 & $1: 2$ & $\mathrm{NaHCO}_{3}$ & $\mathrm{Fe}(\mathrm{acac})_{3}(1.2)$ & 100 & 64 \\
\hline 11 & $1: 2$ & $\mathrm{NaHCO}_{3}$ & $\mathrm{Fe}(\mathrm{acac})_{3}(2.0)$ & 100 & 45 \\
\hline $12^{c}$ & $1: 2$ & $\mathrm{NaHCO}_{3}$ & $\mathrm{Fe}(\mathrm{acac})_{3}(1.2)$ & 100 & 43 \\
\hline
\end{tabular}

${ }^{a}$ Reaction conditions: 1,4-enyne (1a, 0.2 mmol, 1.0 equiv.), 2 ( $w$ equiv.), metal salt ( $x$ equiv.), base (1.0 equiv.) under the air conditions, for $6 \mathrm{~h} .{ }^{b}$ Isolated yield based on 1a. ${ }^{c}$ Under $\mathrm{N}_{2}$ conditions.

\section{2 反应普适性研究}

基于上述优化的反应条件和以苯硅烷 2 为氢源, 对 
1,4-烯炔底物的适用范围进行了研究(表 2). 首先在炔烃 连接的苯环 $\left(\mathrm{R}^{1}\right)$ 的不同位置上导入不同电子性质来考察 对反应的影响. 研究表明: 吸电子基团和供电子基团在 $\mathrm{C}(2) 、 \mathrm{C}(3)$ 和 $\mathrm{C}(4)$ 位置都能顺利参与此反应, 且以 $47 \% \sim 69 \%$ 的产率生成相应的氢炔化产物 3a 3k. 各种 官能团如甲基(1b 和 $1 \mathrm{c})$ 、乙基(1d)、叔丁基(1e)、甲氧 基(1f)、氟 $(1 \mathrm{~g}) 、$ 氯 $(1 \mathrm{~h}, 1 \mathrm{i}$ 和 $1 \mathrm{j})$ 和溴 $(1 \mathrm{k})$ 在优化条件下都 能顺利进行氢自由基引发的3-exo-dig 环化和 1,2-迁移炔 基过程. 2-噻吩基取代的 1,4-烯炔也是有效的底物, 在优
化反应条件下合成了氢炔化产物 31，产率为 59\%。烷基 取代的 1,4-烯炔如叔丁基在最优反应条件下也能进行转 化，尽管相应产物 $3 \mathrm{~m}$ 的收率较低，仅为 $26 \%$. 随后对 1,4-烯炔中非迁移芳基 $\left(\mathrm{R}^{2}\right)$ 的普适性进行了探究. 各种 供电子基如甲基、叔丁基、甲氧基，无论其在对位(1n, 1q 和 1r)、间位 $(10)$ 、邻位 $(1 p)$, 都可很好地适应此反应条 件，生成相应的 1,2-炔基迁移产物 $(\mathbf{3 n} \sim \mathbf{3 r}$ ), 产率为 $55 \% \sim 71 \%$. 在苯环的不同位置上导入各种官能团如氟 $(1 \mathrm{~s})$ 、氯 $(1 \mathrm{t}, 1 \mathrm{u}, 1 \mathrm{v}$ 和 $1 \mathrm{w})$ 和溴 $(1 \mathrm{x})$ 也能顺利进行，以

表 2 氢炔化反应的底物范围

Table 2 Substrate scope of hydroalkynylation

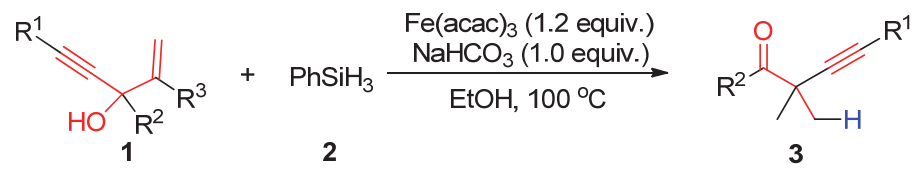

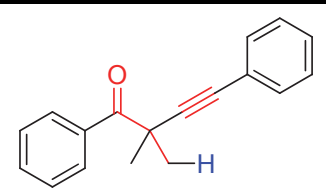

$3 a(64 \%)$

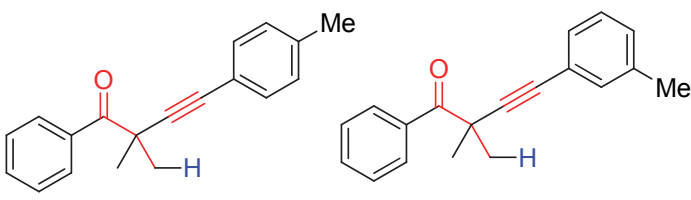

3b $(47 \%)$<smiles>CCCC(C)(C=Cc1ccc(CC)cc1)C(=O)c1ccccc1</smiles>

3d $(51 \%)$<smiles>CCC(C)(C#Cc1ccc(C(C)(C)C)cc1)C(=O)c1ccccc1</smiles>

3 e $(54 \%)$<smiles>COc1ccc(C#CC(C)(C[18OH])C(=O)c2ccccc2)cc1</smiles><smiles>CC(C)(C)CC(C)(C#Cc1ccccc1Cl)C(=O)c1ccccc1</smiles><smiles>CCC(C)(C#Cc1ccc(Br)cc1)C(=O)c1ccccc1</smiles>

3k $(58 \%)$<smiles>CCC(C)(C#Cc1cccs1)C(=O)c1ccccc1</smiles>

3I (59\%)<smiles>CCC(C)(C)C#CC(=O)c1ccccc1</smiles>

$3 \mathrm{~m}(26 \%)$<smiles>CCC(C)(C#Cc1ccccc1)C(=O)c1ccc(C)cc1</smiles>

3n $(55 \%)$<smiles>C=CC#CC(C#Cc1ccccc1)(CC)C(=O)c1cccc([N+](=O)[O-])c1</smiles><smiles>CCC(C#Cc1ccccc1)C(=O)c1ccccc1N</smiles>

$3 p(71 \%)$<smiles>CC=CCCCC(C)C(=O)c1ccc(Br)cc1</smiles>

$3 q(56 \%)$

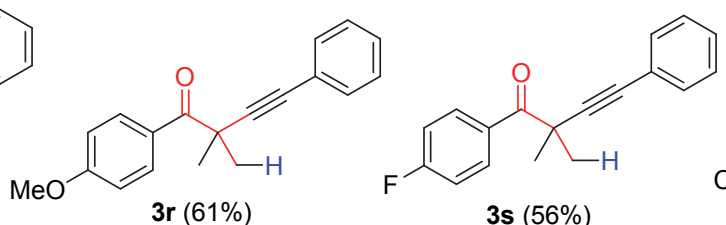<smiles>C/C=C\C=C/C(C)C(=O)c1ccc(Cl)cc1</smiles>

3t $(53 \%)$<smiles>CCCCCCC(=O)c1ccccc1C#CC(C)(C)C(=O)c1ccccc1</smiles><smiles>CCC(C#Cc1ccccc1)C(=O)c1cc(Cl)cc(Cl)c1</smiles><smiles>CCCCC(=O)c1ccc(Br)cc1</smiles><smiles>C/C=C\C=C/c1ccccc1C#CC(C)C(=O)c1ccc2ccccc2c1</smiles>
3y $(50 \%)$<smiles>CC(C)(C#Cc1ccccc1)C(=O)c1cccs1</smiles><smiles>CCC(=O)C(C)C#Cc1ccccc1</smiles>

$3 z(45 \%)$

3aa $(40 \%)$

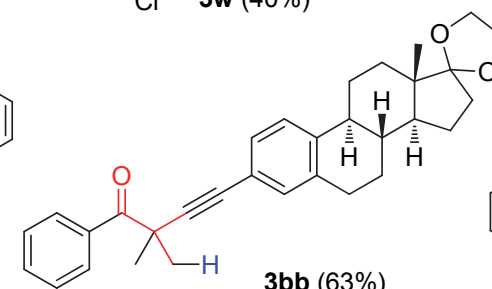<smiles>O=CC(C#Cc1ccccc1)C(=O)c1ccccc1</smiles>

3cc (trace) 
$40 \% \sim 78 \%$ 的收率合成 $\alpha$-炔酮化合物 $3 \mathbf{s} \sim 3 \mathbf{x}$. 值得一提 的是, 空间位阻比较大的 2-萗基衍生物 $1 \mathrm{y}$ 在该反应条 件下也可以 50\%的产率得到 2-萗基取代的 $\alpha$-炔酮化合 物 3y. 除此之外, 噻吩基和乙基取代的 1,4-烯炔 $\mathbf{1 z}$ 和 1aa 经类似的 3-exo-dig 环化和 1,2-炔基迁移过程得到产 物 3z 和 3aa, 收率分别为 $45 \%$ 和 $40 \%$. 雌酮衍生的 1,4烯炔 $1 \mathrm{bb}$ 也能很好地适应此反应条件, 以 $63 \%$ 收率获得 相应的䧳酮衍生物 3bb. 遗憾的是, 将 1,4-烯炔底物中 烯烃连接的甲基变换为氢时, 观测到该反应体系相当复 杂, 仅得到痕量的目标产物 3cc, 且由于其量少而无法 分离得到. 所合成的氢炔化产物 $3 \mathbf{a} \sim \mathbf{3 b b}$ 的结构均经过 核磁共振 ${ }^{1} \mathrm{H} \mathrm{NMR}$ 谱、 ${ }^{13} \mathrm{C} \mathrm{NMR}$ 谱、红外光谱、质谱等 确定. 其中, 产物 $3 \mathrm{e}$ 经过 $\mathrm{X}$ 射线单晶衍射分析进一步确 定了产物结构(图 1) ${ }^{[20]}$.

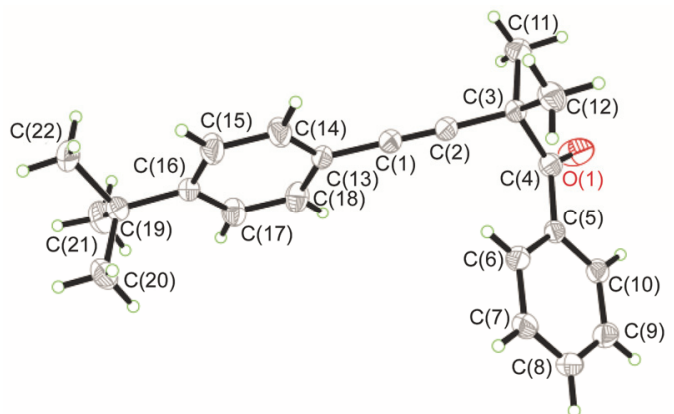

图 $1 \alpha$-炔酮化合物 $3 \mathrm{e}$ 的单晶结构图

Figure 1 X-Ray crystal structure of $\alpha$-alkynyl ketone $3 \mathrm{e}$

\section{3 反应机理}

结合实验结果及文献报道 ${ }^{[10 a, 12,17]}$, 提出了一种可行 的反应途径(Scheme 2). 首先, $\mathrm{Fe}(\mathrm{III})$ 与苯硅烷在乙醇溶 剂中反应产生 $\mathrm{L}_{n} \mathrm{Fe}^{\mathrm{III}} \mathrm{H}$ 物种. 该铁氢物种原位产生的氢 自由基与末端烯烃发生加成反应得到自由基中间体 $\mathbf{A}$, 同时释放出 $\mathrm{Fe}(\mathrm{II})$ 物种. 紧接着, $\mathbf{A}$ 经过 3-exo-dig 环化得 到自由基中间体 $\mathbf{B}$, 经历 $\mathrm{C}-\mathrm{C}$ 键均裂产生更稳定的羟 烷基自由基 $\mathbf{C}$. 随后 $\mathbf{C}$ 在 $\mathrm{Fe}(\mathrm{III})$ 作用下经过单电子转移 过程 $(\mathrm{SET})$ 得到碳正离子 D. 最后 $\mathbf{D}$ 在碱的作用下

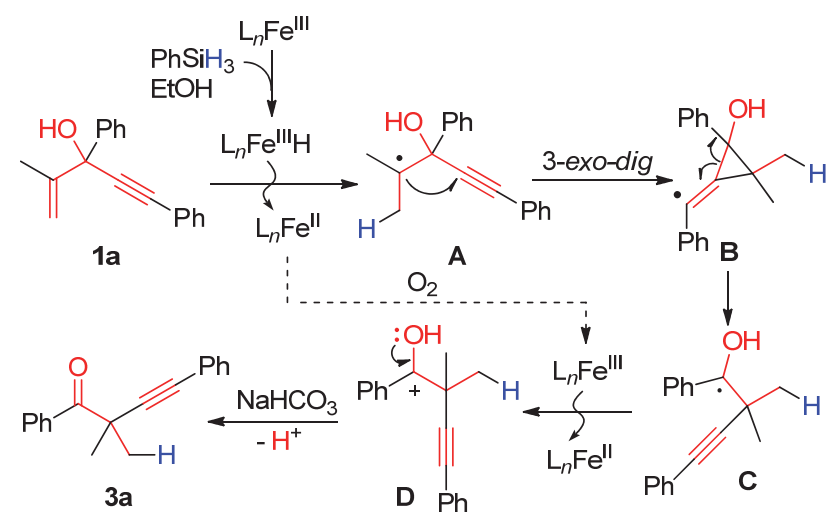

图式 2 可能的反应机理

Scheme 2 Proposed reaction mechanism
去质子化得到氢炔化产物 3a. 在氧气存在下，部分的 $\mathrm{Fe}(\mathrm{II})$ 物种被氧化成 $\mathrm{Fe}(\mathrm{III})$ 物种继续促使该反应的进行. 详细的反应机理目前尚不清晰.

\section{3 结论}

在铁/苯硅烷的催化氢化体系下，利用其原位产生 的氢自由基引发的3-exo-dig环化实现了分子内 1,2-炔基 迁移，以中等到良好的产率合成了一系列 $\alpha$-炔酮化合 物. 此反应进一步证实了自由基诱导的反鲍德温规则 3-exo-dig 环化的可行性. 值得一提的是, 以廉价铁盐为 介导剂和氧化剂以及易得的苯硅烷作为氢源, 通过 1,2炔基迁移实现形式上的非活性烯烃的氢炔化目前尚无 文献先例，因此，为非活性烯烃的氢炔化反应提供了一 种切实可行的、有效的合成策略.

\section{References}

[1] (a) Merino, E.; Nevado, C. Chem. Soc. Rev. 2014, 43, 6598. (b) Tang, S.; Liu, K.; Liu, C.; Lei, A. Chem. Soc. Rev. 2015, 44, 1070. (c) Koike, T.; Akita, M. Acc. Chem. Res. 2016, 49, 1937. (d) Studer, A.; Curran, D. P. Angew. Chem., Int. Ed. 2016, 55, 58. (e) Wu, K.; Liang, Y.; Jiao, N. Molecules 2016, 21, 352. (f) Lan, X.-W.; Wang, N.-X.; Xing, Y. Eur. J. Org. Chem. 2017, 2017, 5821. (g) Zhang, J.-S.; Liu, L.; Chen, T.; Han, L.-B. Chem.-Asian J. 2018, 13, 2277. (h) Zhang, J. G.; Wu, Z. X.; Xie, F.; Zhang, W. B. Chin. J. Org. Chem. 2018, 38, 1319. (张金刚, 吴正兴, 谢芳, 张万斌, 有机化 学, 2018, 38, 1319.) (i) Fu, X. F.; Zhao, W. X. Chin. J. Org. Chem. 2019，39，625. (付晓飞，赵文献，有机化学，2019，39，625.) (j) Yang, W. C.; Qi, X. X.; Chen, P. H.; Liu, G. S. Chin. J. Org. Chem. 2019，39，122. (杨文铖，开晓旭，陈品红，刘国生，有机化学, 2019, 39, 122.) (k) Bian, R. J.; Bao, X. G. Chin. J. Org. Chem. 2017, 37, 190. (市荣剑, 鲍晓光, 有机化学, 2017, 37, 190.) (k) Jiang, B.; Li, J.; Pan, Y.-Y.; Hao, W.-J.; Li, G.; Tu, S.-J. Chin. J. Chem. 2017, 35,323 .

[2] (a) Yin, G.; Mu, X.; Liu, G. Acc. Chem. Res. 2016, 49, 2413. (b) Dhungana, R. K.; Shekhar, K. C.; Basnet, P.; Giri, R. Chem. Rec. 2018, 18, 1314; (c) Koike, T.; Akita, M. Chem 2018, 4, 409. (d) Qiu, G.; Lai, L.; Cheng, J.; Wu, J. Chem. Commun. 2018, 54, 10405. (e) Peng, H. H.; Yuan, Z. L.; Chen, P. H.; Liu, G. S. Chin. J. Chem. 2017, 35, 876. (f) Song, H.; Liu, X. Y.; Qin, Y. Acta Chim. Sinica 2017, 75, 1137. (宋影, 刘小宇, 秦勇, 化学学报, 2017, 75, 1137.)

[3] (a) Wu, X.; Wu, S.; Zhu, C. Tetrahedron Lett. 2018, 59, 1328; (b) Wu, X.; Zhu, C. Chin. J. Chem. 2019, 37, 171; (c) Li, W.; Xu, W.; Xie, J.; Yu, S.; Zhu, C. Chem. Soc. Rev. 2018, 47, 654.

[4] (a) Chen, Z.-M.; Bai, W.; Wang, S.-H.; Yang, B.-M.; Tu, Y.-Q.; Zhang, F.-M. Angew. Chem., Int. Ed. 2013, 52, 9781. (b) Liu, X.; Xiong, F.; Huang, X.; Xu, L.; Li, P.; Wu, X. Angew. Chem., Int. Ed. 2013, 52, 6962. (c) Egami, H.; Shimizu, R.; Usui, Y.; Sodeoka, M. Chem. Commun. 2013, 49, 7346. (d) Chen, Z.-M.; Zhang, Z.; Tu, Y.-Q.; Xu, M.-H.; Zhang, F.-M.; Li, C.-C.; Wang, S.-H. Chem. Commun. 2014, 50, 10805. (e) Chu, X.-Q.; Zi, Y.; Meng, H.; Xu, X.-P.; Ji, S.-J. Chem. Commun. 2014, 50, 7642. (f) Mi, X.; Wang, C.; Huang, M.; Wu, Y.; Wu, Y. Org. Biomol. Chem. 2014, 12, 8394. (g) Chu, X.-Q.; Meng, H.; Zi, Y.; Xu, X.-P.; Ji, S.-J. Chem. Commun. 2014, 50, 9718. (h) Li, Y.; Liu, B.; Li, H.-B.; Wang, Q.; Li, J.-H. Chem. Commun. 2015, 51, 1024. (i) Song, R.-J.; Tu, Y.-Q.; Zhu, D.-Y.; Zhang, F.-M.; Wang, S.-H. Chem. Commun. 2015, 51, 749. (j) Zhao, J.; Fang, H.; Song, R.; Zhou, J.; Han, J.; Pan, Y. Chem. Commun. 2015, 51, 599.

[5] (a) Wu, Z.; Ren, R.; Zhu, C. Angew. Chem., Int. Ed. 2016, 55, 10821. (b) Wang, N.; Li, L.; Li, Z.-L.; Yang, N.-Y.; Guo, Z.; Zhang, H.-X.; Liu, X.-Y. Org. Lett. 2016, 18, 6026. (c) Ji, M.; Wu, Z.; Yu, J.; Wan, X.; Zhu, C. Adv. Synth. Catal. 2017, 359, 1959. (d) Ren, R.; Wu, Z.; Huan, L.; Zhu, C. Adv. Synth. Catal. 2017, 359, 3052.

[6] (a) Yu, J.; Wang, D.; Xu, Y.; Wu, Z.; Zhu, C. Adv. Synth. Catal. 2018, 360, 744. (b) Chen, D.; Ji, M.; Yao, Y.; Zhu, C. Acta Chim. 
Sinica 2018，76，951. (陈栋, 吉梅山, 姚英明, 朱晨, 化学学报, 2018, 76, 951.) (c) Chen, D.; Wu, Z.; Yao, Y.; Zhu, C. Org. Chem. Front. 2018, 5, 2370. (d) Wang, N.; Wang, J.; Guo, Y.-L.; Li, L.; Sun, Y.; Li, Z.; Zhang, H.-X.; Guo, Z.; Li, Z.-L.; Liu, X.-Y. Chem. Commun. 2018, 54, 8885.

[7] Li, Z. L.; Li, X. H.; Wang, N.; Yang, N. Y.; Liu, X. Y. Angew. Chem., Int. Ed. 2016, 55, 15100 .

[8] (a) Wu, Z.; Wang, D.; Liu, Y.; Huan, L.; Zhu, C. J. Am. Chem. Soc. 2017, 139, 1388. (b) Gu, L. J.; Gao, Y.; Ai, X. H.; Jin, C.; He, Y. H.; Li, G. P.; Yuan, M. L. Chem. Commun. 2017, 53, 12946. (c) He, Y.; Wang, Y.; Gao, J.; Zeng, L.; Li, S.; Wang, W.; Zheng, X.; Zhang, S.; Gu, L.; Li, G. Chem. Commun. 2018, 54, 7499. (d) Wang, H.; Xu, Q.; Yu, S. Org. Chem. Front. 2018, 5, 2224. (e) Wang, M.; Wu, Z.; Zhang, B.; Zhu, C. Org. Chem. Front. 2018, 5, 1896. (f) Wei, X.-J.; Noël, T. J. Org. Chem. 2018, 83, 11377. (g) Zhang, H.; Wu, X.; Zhao, Q.; Zhu, C. Chem.-Asian J. 2018, 13, 2453. (h) Zhang, W.; Zou, Z.; Wang, Y.; Wang, Y.; Liang, Y.; Wu, Z.; Zheng, Y.; Pan, Y. Angew. Chem., Int. Ed. 2019, 58, 624. (i) Zheng, M.-W.; Yuan, X.; Cui, Y.-S.; Qiu, J.-K.; Li, G.; Guo, K. Org. Lett. 2018, $20,7784$.

[9] (a) Tang, X.; Studer, A. Angew. Chem., Int. Ed. 2018, 57, 814. (b) Gao, Y. Y.; Mei, H. B.; Han, J. L.; Pan, Y. Chem.-Eur. J. 2018, 24, 17205.

[10] (a) Xu, Y.; Wu, Z.; Jiang, J.; Ke, Z.; Zhu, C. Angew. Chem., Int. Ed. 2017, 56, 4545. (b) Tang, X.; Studer, A. Chem. Sci. 2017, 8, 6888. (c) Liu, J.; Li, W. P.; Xie, J.; Zhu, C. J. Org. Chem. Front. 2018, 5, 797.

[11] (a) Campbell, M. J.; Pohlhaus, P. D.; Min, G.; Ohmatsu, K.; Johnson, J. S. J. Am. Chem. Soc. 2008, 130, 9180. (b) Alabugin, I. V.; Gilmore, K.; Manoharan, M. J. Am. Chem. Soc. 2011, 133, 12608.

[12] Zhao, Q.; Ji, X.-S.; Gao, Y.-Y.; Hao, W.-J.; Zhang, K.-Y.; Tu, S.-J.; Jiang, B. Org. Lett. 2018, 20, 3596.

[13] (a) Wang, Z.-X.; Bai, X.-Y.; Li, B.-J. Synlett 2017, 28, 509. (b) Yang, X.; Yang, W.; Deng, Y. Y.; Yang, D. Q. Chin. J. Org. Chem. 2017, 37, 2512. (杨新, 杨文, 邓颖颖, 杨定乔, 有机化学, 2017, 37, 2512.)

[14] (a) Knöpfel, T. F.; Carreira, E. M. J. Am. Chem. Soc. 2003, 125, 6054. (b) Nishimura, T.; Guo, X.-X.; Uchiyama, N.; Katoh, T.; Hayashi, T. J. Am. Chem. Soc. 2008, 130, 1576. (c) Shirakura, M.; Suginome, M. J. Am. Chem. Soc. 2008, 130, 5410. (d) Fillion, E.; Zorzitto, A. K. J. Am. Chem. Soc. 2009, 131, 14608. (e) Nishimura, T.; Sawano, T.; Hayashi, T. Angew. Chem., Int. Ed. 2009, 48, 8057. (f) Nishimura, T.; Tokuji, S.; Sawano, T.; Hayashi, T. Org. Lett. 2009, 11, 3222. (g) Shirakura, M.; Suginome, M. Org. Lett. 2009, 11, 523. (h) Villarino, L.; López, F.; Castedo, L.; Mascareñas, J. L.
Chem.-Eur. J. 2009, 15, 13308. (i) Yazaki, R.; Kumagai, N.; Shibasaki, M. J. Am. Chem. Soc. 2010, 132, 10275. (j) Sawano, T.; Ashouri, A.; Nishimura, T.; Hayashi, T. J. Am. Chem. Soc. 2012, 134, 18936. (k) Dou, X.; Huang, Y.; Hayashi, T. Angew. Chem., Int. Ed. 2016, 55, 1133. (1) Hosseyni, S.; Smith, C. A.; Shi, X. Org. Lett. 2016, 18, 6336. (m) Fan, B.-M.; Yang, Q.-J.; Hu, J.; Fan, C.-L.; Li, S.-F.; Yu, L.; Huang, C.; Tsang, W. W.; Kwong, F. Y. Angew. Chem., Int. Ed. 2012, 51, 7821. (n) Chen, H. L.; Li, S. F.; Xu, J. B.; Yang, Q. J.; Liu, S. S.; Zhou, Y. Y.; Huang, C.; Fan, B. M. Acta Chim. Sinica 2013, 71, 1243. (陈花䂞, 李嗣锋, 徐建斌, 杨清镜, 刘珊珊, 周永 云, 黄超, 樊保敏, 化学学报, 2013, 71, 1243.) (o) Fan, B.; Li, S.; Chen, H.; Lu, Z.; Liu, S.; Yang, Q.; Yu, L.; Xu, J.; Zhou, Y.; Wang, J. Adv. Synth. Catal. 2013, 355, 2827.

[15] (a) Bai, X.-Y.; Wang, Z.-X.; Li, B.-J. Angew. Chem., Int. Ed. 2016, 55, 9007. (b) Bai, X.-Y.; Zhang, W.-W.; Li, Q.; Li, B.-J. J. Am. Chem. Soc. 2018, 140, 506. (c) Chen, Y.; Wang, Z.-X.; Li, Q.; Xu, L.-J.; Li, B.-J. Org. Chem. Front. 2018, 5, 1815.

[16] (a) Nishimura, T.; Katoh, T.; Takatsu, K.; Shintani, R.; Hayashi, T. J. Am. Chem. Soc. 2007, 129, 14158. (b) Shirakura, M.; Suginome, M. J. Am. Chem. Soc. 2009, 131, 5060. (c) Canterbury, D. P.; Micalizio, G. C. J. Am. Chem. Soc. 2010, 132, 7602. (d) Avocetien, K. F.; Li, J. J.; Liu, X.; Wang, Y.; Xing, Y.; O’Doherty, G. A. Org. Lett. 2016, 18, 4970. (e) Teng, H.-L.; Ma, Y.; Zhan, G.; Nishiura, M.; Hou, Z. ACS Catal. 2018, 8, 4705.

[17] Lo, J. C. L.; Gui, J. H.; Yabe, Y. K.; Pan, C. M.; Baran, P. S. Nature 2014, 516, 343

[18] (a) Lo, J. C.; Yabe, Y.; Baran, P. S. J. Am. Chem. Soc. 2014, 136, 1304. (b) Gui, J. H.; Pan, C. M.; Jin, Y.; Qin, T.; Lo, J. C.; Lee, B. J.; Spergel, S. H.; Mertzman, M. E.; Pitts, W. J.; La Cruz, T. E.; Schmidt, M. A.; Darvatkar, N.; Natarajan, S. R.; Baran, P. S. Science 2015, 348, 886. (c) Dao, H. T.; Li, C.; Michaudel, Q.; Maxwell, B. D.; Baran, P. S. J. Am. Chem. Soc. 2015, 137, 8046. (d) Zheng, J.; Wang, D.; Cui, S. Org. Lett. 2015, 17, 4572. (e) Zheng, J.; Qi, J.; Cui, S. Org. Lett. 2016, 18, 128. (f) Shen, Y.; Qi, J.; Mao, Z.; Cui, S. Org. Lett. 2016, 18, 2722. (g) Qi, J.; Zheng, J.; Cui, S. Org. Chem. Front. 2018, 5, 222. (h) Qi, J.; Zheng, J.; Cui, S. Org. Lett. 2018, 20 , 1355. (i) Deng, Z.; Chen, C.; Cui, S. RSC Adv. 2016, 6, 93753.

[19] Shen, Y.; Huang, B.; Zheng, J.; Lin, C.; Liu, Y.; Cui, S. Org. Lett. 2017, 19, 1744.

[20] CCDC 1913020 (3e) contains the supplementary crystallographic data for this paper. These data can be obtained free of charge from The Cambridge Crystallographic Data Centre via www. ccdc. cam. ac. uk/data_request/cif.

(Zhao, C.) 